\title{
Visualization of Hysteresis Phenomenon of Shock Waves in Supersonic Internal Flow
}

\author{
Abhilash Suryan*, Choon Sik Shin*, Toshiaki Setoguchi** and Heuy Dong Kim ${ }^{\dagger}$
}

\begin{abstract}
Hysteresis is an effect by which the order of previous events influences the order of subsequent events. Hysteresis phenomenon of supersonic internal flows with shock waves has not yet been clarified satisfactorily. In the present study, experiments are carried out on internal flow in a supersonic nozzle to clarify the hysteresis phenomena for the shock waves. Flow visualization is carried out separately on the straight and divergent channels downstream of the nozzle throat section. Results obtained were compared with numerically simulated data. The results confirmed hysteresis phenomenon for shock wave in the Laval nozzle at a certain specific condition. The relationship between hysteresis phenomenon and the range of the rate of change of pressure ratio with time was shown experimentally. The existence of hysteretic behavior in the formation, both the location and strength, of shock wave in the straight part of the supersonic nozzle with a range of pressure ratio has also been confirmed numerically.
\end{abstract}

Key Words: Internal flow, Hysteresis, Laval nozzle, Shock wave, Supersonic flow

\section{NOMENCLATURE}

$\mathrm{C}_{\mathrm{p}} \quad$ specific heat at constant pressure $[\mathrm{J} / \mathrm{kg}-\mathrm{K}]$

$D_{\text {e }} \quad$ nozzle exit diameter, characteristics length [mm]

$D_{t}$ diameter at nozzle throat, characteristics length [mm]

$E$ total energy per unit mass $[\mathrm{J} / \mathrm{kg}]$

$\boldsymbol{F}$ inviscid flux vectors

$\boldsymbol{G}$ viscous flux vectors

$H \quad$ total enthalpy per unit mass $[\mathrm{J} / \mathrm{kg}]$

$i \quad$ unit vector in the direction of the $x$-axis

$j \quad$ unit vector in the direction of the $y$-axis

$k \quad$ unit vector in the direction of the $z$-axis

$L \quad$ location of Mach disk [mm]

$p \quad$ static pressure $[\mathrm{Pa}]$

$\boldsymbol{Q}$ dependent vector of primary variables

$t$ time [s]

$T$ temperature [K]

$U_{\mathrm{r}}$ reference velocity $[\mathrm{m} / \mathrm{s}]$

'School of Mechanical Engineering, Andong National University

E-mail: kimhd@andong.ac.kr

**Institute of Ocean Energy, Saga University, Japan $v_{x}$ Cartesian velocity component in $x$-direction $[\mathrm{m} / \mathrm{s}]$

$v_{y}$ Cartesian velocity component in $y$-direction $[\mathrm{m} / \mathrm{s}]$

$v_{z} \quad$ Cartesian velocity component in $z$-direction $[\mathrm{m} / \mathrm{s}]$

$\phi \quad$ pressure ratio $p_{0} / p_{\mathrm{b}}$

$\rho$ density $\left[\mathrm{kg} / \mathrm{m}^{3}\right]$

$\tau \quad$ shear stress $[\mathrm{Pa}]$

$\begin{array}{ll}\text { Subscripts } \\ 0 & \text { stagnation } \\ b & \text { back } \\ x & x \text {-coordinate } \\ y & y \text {-coordinate } \\ z & z \text {-coordinate }\end{array}$

\section{Introduction}

Hysteresis in general may be defined as the phenomenon of the lagging of an effect behind its cause or in other words an effect by which the order of previous events influences the order of subsequent events. In this phenomenon two (or more) physical quantities bear a relationship which depends on prior history. Hysteresis was initially thought off as a problematic phenomenon, but is 
now widely recognized as significant in engineering and technology.

Hysteresis phenomena in fluid flow systems have become a topic of research off late for the large variety of applications in which they are encountered. This phenomenon is well known for external flows such as reflection of shock waves in supersonic jets. Researchers have already clarified that when the high-pressure gas is exhausted to atmosphere from the nozzle exit, the Mach disk is formed in the expanded supersonic jet at a specific condition. The jet structure is a well known fundamental phenomenon in supersonic fluid mechanics. This type of jet is very important for some industrial devices ${ }^{(1,2)}$. Teshima $^{(3)}$ has suggested the possibility for hysteresis in the supersonic jet formed by a rectangular orifice. Chpoun and Ben-Dor ${ }^{(4)}$ numerically confirmed the hysteresis phenomenon in the regular to the Mach reflection transition in steady flows. Since then many researchers have proven the existence of hysteresis phenomenon in the interference form of the shock wave in the flow.

On the interference form of oblique shock wave of two-dimensional flow, the hysteresis phenomenon on the mutual transition of the regular reflection and Mach reflection is mainly reported from the relationship between Mach number and incidence shock wave angle of the flow ${ }^{(4,5,6,7,8)}$. Gribben et al. ${ }^{(9)}$ have shown for the circular jet that the hysteresis exits on the relation between the location of Mach disk and pressure ratio, and this phenomenon is influenced by the time history effect of plume development. Recent studies reported that in two-dimensional supersonic jet, the hysteresis phenomenon for the reflection type of shock wave occurred under the quasi-steady flow of jet and the transitional pressure ratio between the regular reflection and Mach reflection is affected by this phenomenon ${ }^{(10,11,12)}$. Irie et al. ${ }^{(13)}$, observed such hysteresis phenomenon of the under expanded dry air jet is produced during the transient processes of jet pressure ratio, in which the jet flow obtained in the startup transient is different from that in the shutdown transient. Kim et al. ${ }^{(14)}$, have investigated the effect of nonequilibrium condensation on hysteresis phenomenon of under expanded moist jets and found that the under-expanded moist air jet leads to less hysteresis of the jet, compared with the dry air jets. However, the hysteresis phenomenon of a supersonic internal flow for shock waves is yet to be clarified satisfactorily. From previous experimental and numerical studies, it is understood that the shock wave exist in the supersonic part of a Laval nozzle and in some cases normal shock is also formed which results a sudden rise in temperature across the shock. The nonhomogeneous temperature increase across the shock, caused by the boundary layer thickening ahead of the shock and the resulting precompression prevents the quasi 1-D evolution of the flow downstream ${ }^{(15)}$. Additionally, due to multiple boundary layer interactions the single shock disintegrates into a so called pseudo-shock system; i.e., into a sequence of periodic weak compression and expansion regions ${ }^{(16)}$. On increasing the nozzle pressure ratio, the shock position is moved towards the nozzle exit. The occurrence of the reverse of this phenomenon is also true. Therefore, it calls for experimental as well as numerical investigation on the hysteresis phenomena for the shock wave in a supersonic nozzle.

The objective of the present experimental study is to clarify the hysteresis phenomena for the shock wave in a supersonic nozzle. Relationship between hysteresis phenomenon and rate of the change of pressure ratio with time is also discussed. Influence of the hysteresis phenomena on the location of shock wave in the nozzle was also studied. The results obtained were compared with numerically simulated data.

\section{Experimental Work}

The schematic diagram of the experimental apparatus is described in Fig. 1. The apparatus consisted of an air compressor, air drier, air reservoir, electronic control valve, plenum chamber and the nozzle. Plenum chamber is placed upstream of the nozzle. The test section is placed downstream of the nozzle throat and optical glass windows are installed on both the side walls of the test section for flow visualization.

Fig. 2 shows the detailed nozzle configuration 


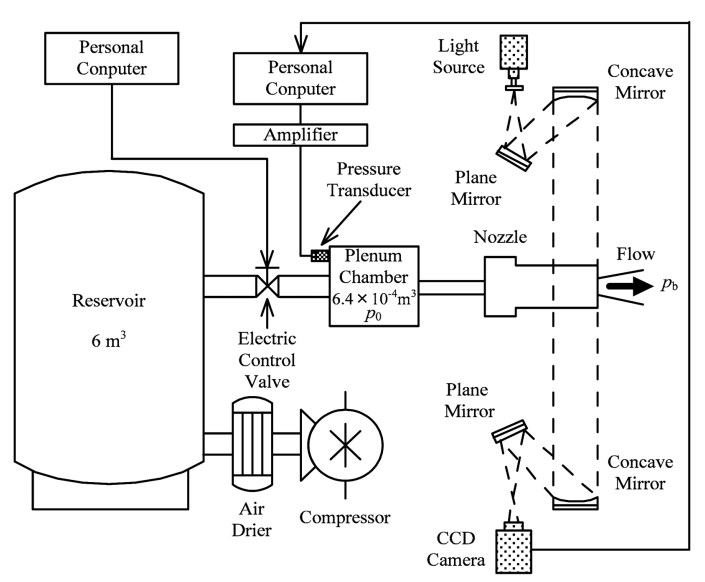

Fig. 1. Schematic Diagram for the experimental setup

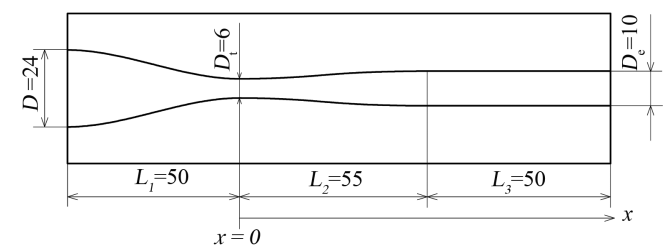

Fig. 2. Nozzle geometry (all dimensions in $\mathrm{mm}$ )

used in the present work. The nozzle was designed using the method of characteristics. The design Mach number of supersonic nozzle is $M_{\mathrm{e}}=2.0$ with the throat and exit diameters of $D_{\mathrm{t}}=6.0$ and $D_{\mathrm{e}}=10.0 \mathrm{~mm}$, respectively.

In the present experiments, pressure ratio was continuously changed with time using the electronic control valve. The symbol, $p_{0}$ and $p_{\mathrm{b}}$ represent the stagnation pressure of the plenum chamber and back pressure (atmospheric pressure), respectively. For shock wave in the straight part of the nozzle (Case 1), the range of pressure ratio was from 2.16 to 2.86 . For shock wave in the divergent part (Case 2), it is from 1.42 to 2.02. The rate of the change of pressure ratio with time is from $0.107(1 / \mathrm{s})$ to $0.437(1 / \mathrm{s})$ for Case 1 and from 0.112 $(1 / \mathrm{s})$ to $0.338(1 / \mathrm{s})$ for Case 2 .

Compressed dry air is discharged from nozzle exit through the plenum chamber. The flow field was investigated by Schlieren technique. Visualization and measurement of pressure ratio were conducted simultaneously. The location of the first shock wave $L$ was obtained from Schlieren pictures.

In the present experimental conditions, it is found that the time delay exists for response of change of flow to change of pressure ratio at $\Delta \dot{\phi}>$ $0.229(1 / \mathrm{s})$ for Case 1 and $\Delta \dot{\phi}>0.338(1 / \mathrm{s})$ for Case 2. The results were determined from the change in position of the shock wave in the nozzle.

\section{Numerical Simulation}

\subsection{Numerical Methods}

Computational Fluid Dynamics simulations were carried out under conditions that replicate the experimental investigations of hysteresis phenomena of shock waves in the straight part of the nozzle. The flow under study was treated as compressible, viscous, unsteady and turbulent. The governing equations are given by the conservation forms of mass, momentum and energy. The axisymmetric, mass averaged, time-dependent Navier-Stokes equations, with the two-equation $k-\omega \mathrm{SST}$ (ShearStress Transport) turbulent model ${ }^{(17,18,19)}$ are employed in the present computation.

$$
\Gamma \frac{\partial}{\partial t} \int_{v} \boldsymbol{Q} d A+\oint[\boldsymbol{F}-\boldsymbol{G}] d A=0
$$

where $\boldsymbol{F}$ and $\boldsymbol{G}$ are the inviscid and viscous flux vectors in standard conservation form, and $\boldsymbol{Q}$ is the dependent vector of primary variables.

$$
\begin{aligned}
& \boldsymbol{F}=\left[\rho v, \rho v v_{x}+p \hat{i}, \rho v v_{y}+p \hat{j}, \rho v v_{z}+p \hat{k}, \rho v H\right]^{T} \\
& \boldsymbol{G}=\left[0, \tau_{x i}, \tau_{y i}, \tau_{z i}, \tau_{i j} v_{j}+q\right]^{T} \\
& \boldsymbol{Q}=\left[p, v_{x}, v_{y}, v_{z}, T\right]^{T}
\end{aligned}
$$

where $H$ is total enthalpy per unit mass and is related to the total energy $E$ by;

$$
H=E+p / \rho,
$$

where $E$ includes both internal energy and kinetic energy. The preconditioning matrix $\Gamma$ is included in Eq. (1) to provide an efficient solution of the present axisymmetric compressible flow. This matrix is given by; 


$$
\Gamma=\left[\begin{array}{ccccc}
\theta & 0 & 0 & 0 & \rho_{T} \\
\theta v_{x} & \rho & 0 & 0 & \rho_{T} v_{x} \\
\theta v_{y} & 0 & \rho & 0 & \rho_{T} v_{y} \\
\theta v_{z} & 0 & 0 & \rho & \rho_{T} v_{x} \\
\theta H & \rho v_{x} & \rho v_{y} & \rho v_{z} & \rho_{T} H+\rho C_{p}
\end{array}\right]
$$

where $\rho_{T}$ is the derivative of density with respect to temperature at constant pressure. The parameter $\theta$ is defined as;

$$
\theta=\frac{1}{U_{r}^{2}}-\left(\rho_{T} H+\rho C_{p}\right)
$$

In Eq. (4), the reference velocity $U_{r}$ is chosen such that the Eigen values of the system remain well conditioned with respect to the convective and diffusive timescales and $C_{p}$ is the specific heat at constant pressure.

The preconditioned governing equations are discretized spatially using a Finite volume scheme, in which the physical domain is subdivided into numerical cells and the integral equations are applied to each cell. Also, for the time derivatives in the governing equations, an implicit multistage time stepping scheme, which is advanced from time $t$ to time $t+\Delta t$ with a 2 nd order Euler backward scheme for physical time and implicit pseudo-time marching scheme for inner iteration, is used.

The computational domain and boundary conditions for simulating the hysteresis phenomena in the straight part of the Laval nozzle in the present study are illustrated in Fig. 3. Axisymmetric geometry is considered. The boundary conditions used are the inlet total pressure and the outlet static pressure respectively. At the walls, adiabatic no-slip conditions are applied. In order to ensure domain independent solutions, the upstream domain in the present computations extends

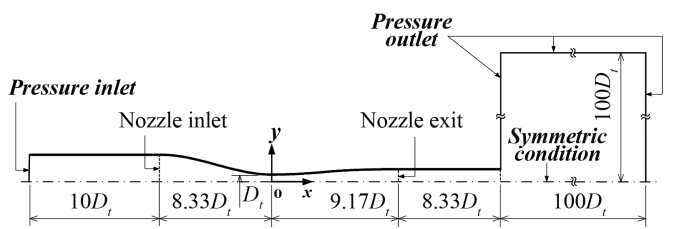

Fig. 3. Computational domain and the boundary conditions straight to the distance of $10 D_{\mathrm{t}}$ upstream from the nozzle inlet, and the downstream extends straight to $8.33 D_{\mathrm{t}}$ from the nozzle exit and then extends again to the distance of $100 D_{\mathrm{t}}$ both in the $x$ - and $y$ directions, respectively.

A structured grid system was employed in the computations. The fineness of the computational grids was examined to ensure that the obtained solutions were grid independent. The grids were densely clustered in the near wall regions to capture the flow features in the boundary layers. A solution convergence was obtained when the residuals for each of the conserved variables were reduced to below the order of magnitude 4 . The net mass flux was also checked and there was only an allowable imbalance through the computational boundaries.

\subsection{Computational Procedure}

Flow fields involving hysteresis cycles had been computed by numerical simulations ${ }^{(20)}$, in which the flow boundary conditions are systematically changed to obtain each of the quasi-steady solutions. Fig. 4 shows the computational procedure for the process of the startup transient, in which the pressure ratio is increased.

The steady supersonic Laval nozzle flow of $\phi=\phi_{\text {st }}$ is computed and the resulting solutions are used as the initial conditions for the first step of the process of the startup transient. In the second step, the pressure ratio is increased by $\Delta \phi$ and the computation is repeated until the transient process is completed, thus leading to a quasi-steady state, as indicated by the black circle. The computed

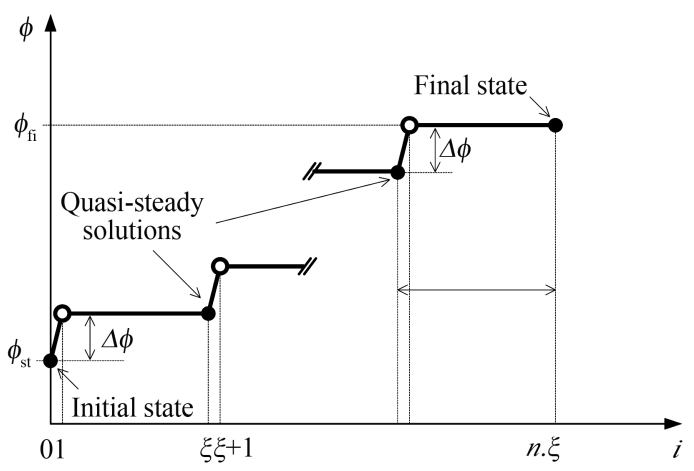

Fig. 4. Computational procedure for the simulation of start-up and shutdown transients 
quasi-steady solutions are used again as the initial conditions for the next step. Consequently the final quasi-steady solutions are obtained for the pressure ratio of $\phi_{\mathrm{fi}}$.

Conversely for the shutdown transients, wherein the pressure ratio is decreased; the final quasisteady solutions are used as the initial conditions. For reference, in the present study, $\phi_{\mathrm{st}}=2.17$, $\phi_{\mathrm{fi}}=3.02, \Delta \phi=0.029$ and $\xi=2000$. Through such a series of computations, the quasi-steady solutions obtained during the startup and shutdown transients are compared to investigate the hysteretic behavior of shock waves generated in the straight part of the supersonic nozzle.

\section{Results and Discussions}

\subsection{Experimental Results}

The Schlieren photographs of the flow fields for straight channel (Case 1) and divergent channel (Case 2) are given by Fig. 5 and 6 respectively. Values of rate of change of the pressure ratio with time $\Delta \dot{\phi}$ for the straight channel and divergent channel are $0.131(1 / \mathrm{s})$ and $0.138(1 / \mathrm{s})$. In both figures, the left side sequence represents the increasing process of pressure ratio and the right side represents the decreasing process of pressure ratio. As seen from these figures, there are differences between the locations of the first shock wave even at the same pressure ratio.

Fig. 7 shows the effect of $\Delta \dot{\phi}$ on the location of the first shock wave $L / D_{\mathrm{t}}$, measured from the nozzle throat, in the range from $0.107(1 / \mathrm{s})$ to $0.331(1 / \mathrm{s})$. As evident from figure, hysteresis loops exist at the course between $\mathrm{A}$ and $\mathrm{B}$. Processes of variation between A and B follow the same course below $\Delta \dot{\phi}=0.289(1 / \mathrm{s})$.

Similarly, for Case 2, the experimental results explaining the effect of the rate of change of pressure ratio with time, $\Delta \dot{\phi}$ on the location of the first shock wave $L / D_{\mathrm{t}}$ is shown in Fig. 8. Here, $\Delta \dot{\phi}$ is range from $0.112(1 / \mathrm{s})$ to $0.338(1 / \mathrm{s})$. Here too hysteresis loops exist at the course between $\mathrm{A}$ and B. Processes of variation between A and B follow the same course below $\Delta \dot{\phi}=0.318(1 / \mathrm{s})$.

Fig. 9(a) and (b) show the relationships between $L / D_{\mathrm{t}}$ and pressure ratio $\phi$ in case of occurrence of hysteresis phenomena for Case $1(\Delta \dot{\phi}=0.131(1 /$

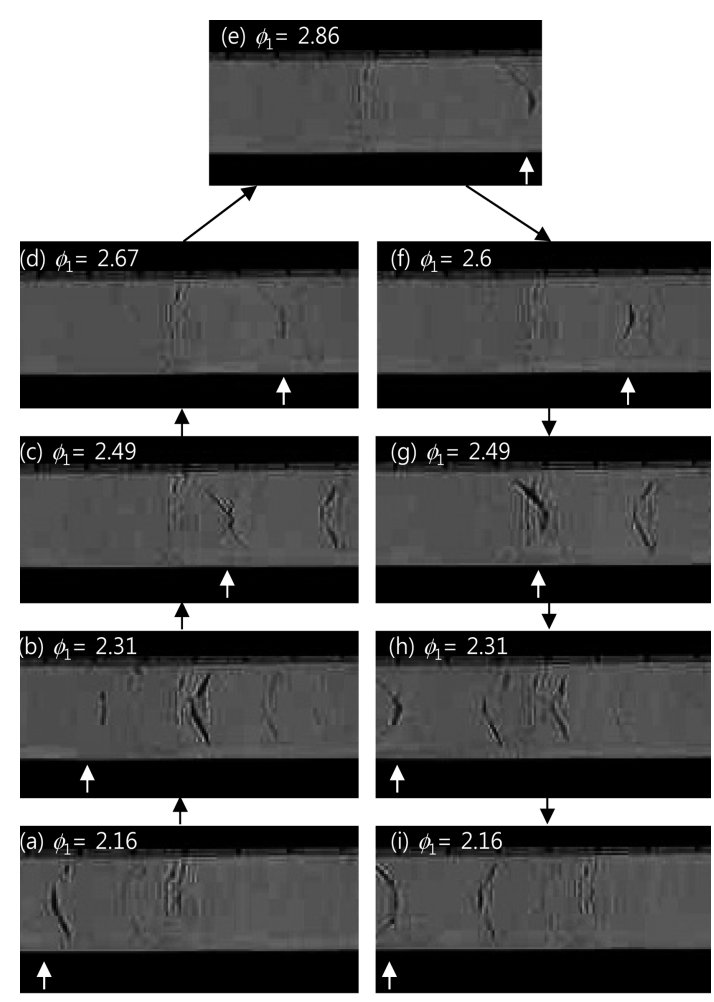

Fig. 5. Schlieren photographs in straight channel

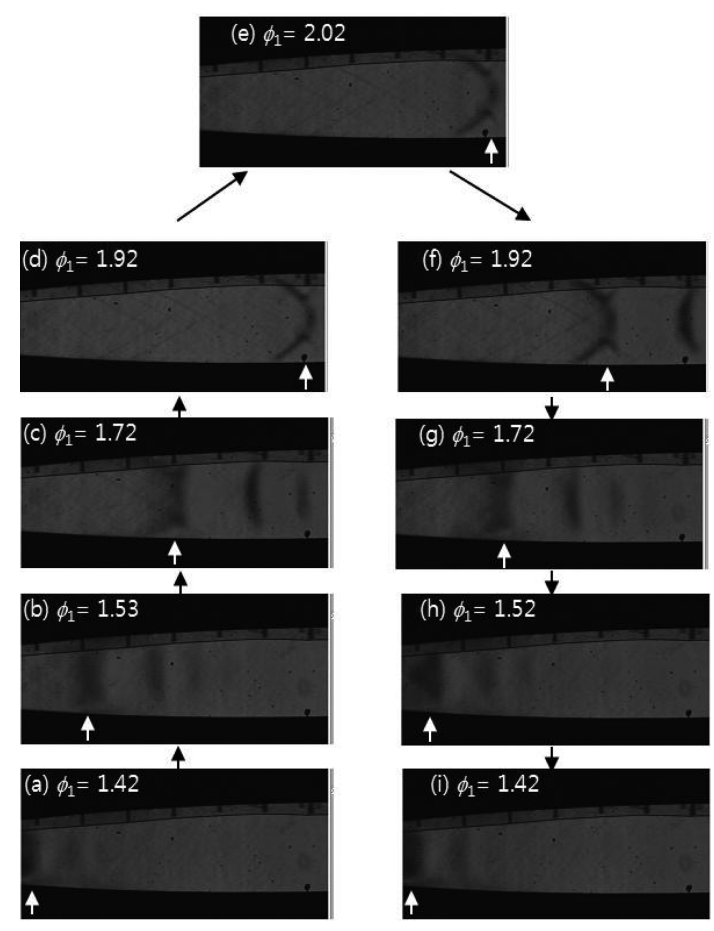

Fig. 6. Schlieren photographs in divergent channel 


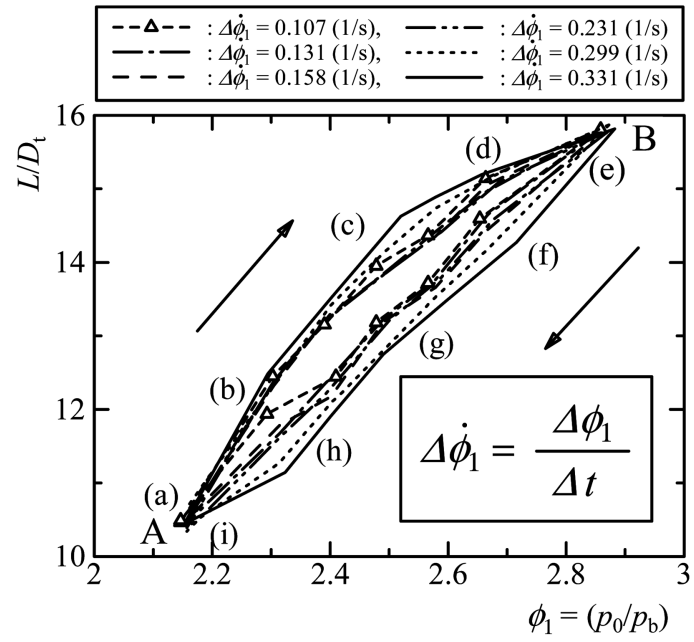

Fig. 7. Effect of rate of change of pressure ratio in straight channel

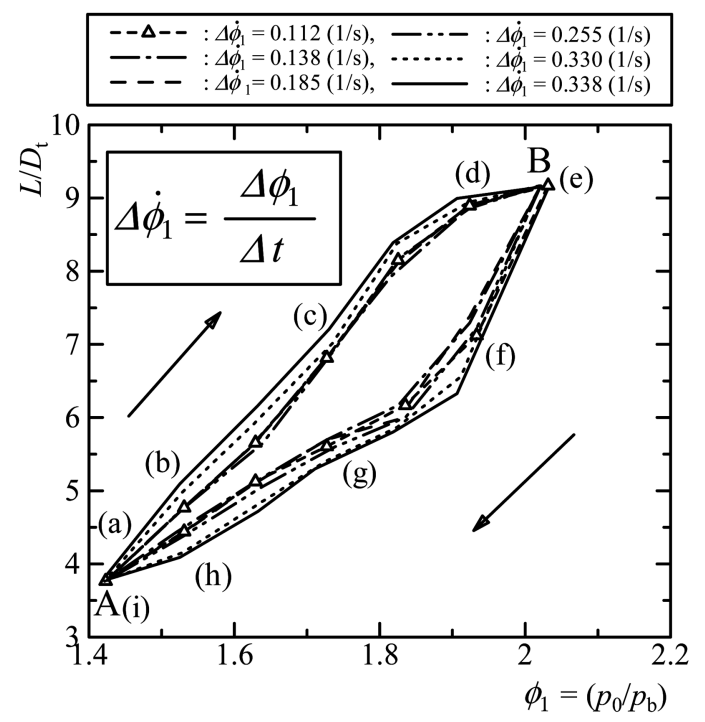

Fig. 8. Effect of rate of change of pressure ratio in divergent channel

s)) and Case $2(\Delta \dot{\phi}=0.138(1 / \mathrm{s}))$, respectively. In both figures, symbols from (a) to (i) correspond to those in Figs. 5 and 6 . As evident from these figures, the existence of two values of $L / D_{\mathrm{t}}$ is confirmed in the ranges of $\phi=2.16-2.86$ for Case 1 and $\phi=1.42-2.02$ for Case 2

In our previous investigations on supersonic jet, the maximum mass flow rate in the jet within a certain pressure ratio changes with pressure ratio

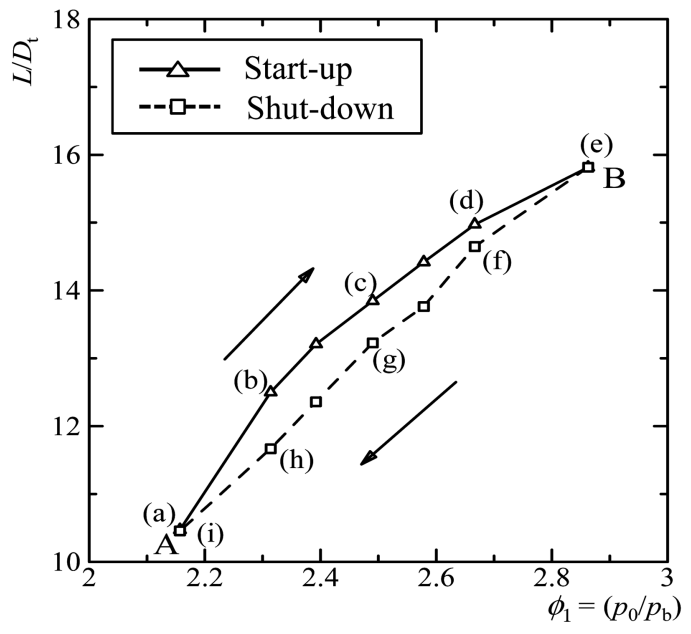

(a) Straight channel

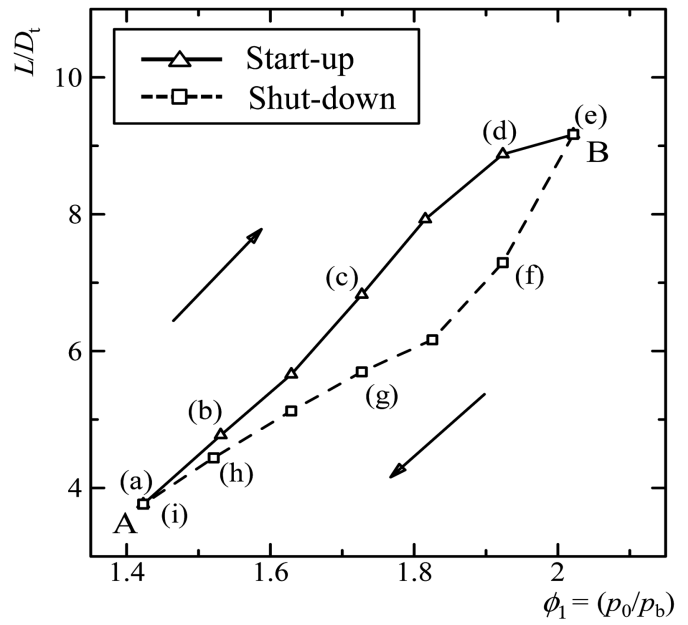

(b) Divergent channel

Fig. 9. Hysteretic behavior in the location of Mach disk

and this condition is in tune with the range of occurrence of the hysteresis phenomenon. From this result, it is considered that the mass flow rate is related to the hysteresis loop generation mechanism. However, as the flow has a nonlinear nature, the flow field is changed by influence of initial state of the entire flow field. Therefore, in order to understand the phenomenon, the flow inside and outside the nozzle should be considered.

\subsection{Computational Results}

In order to confirm the existence of hysteresis phenomena for shock wave in the supersonic nozzle, a computational study is conducted in a 


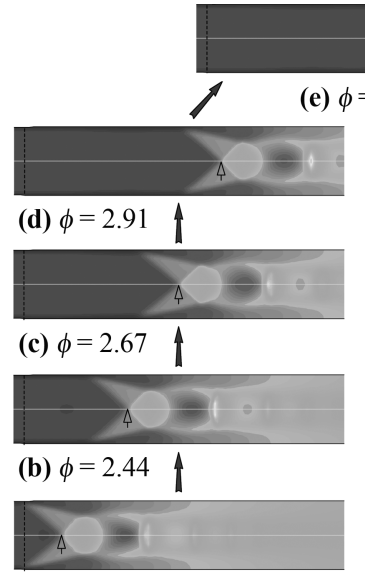

(a) $\phi=2.20$

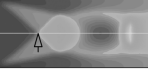

(e) $\phi=3.02$
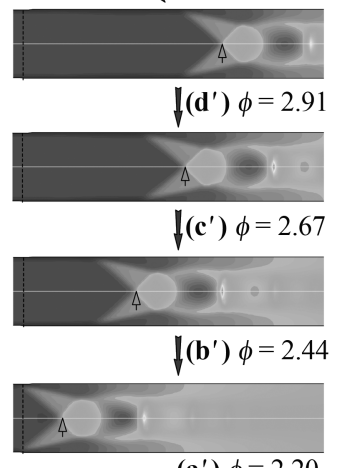

$\left(\mathbf{a}^{\prime}\right) \phi=2.20$

Fig. 10. Density contours illustrating hysteresis phenomena in the straight channel

range of pressure ratio from $\phi=2.17$ to 3.02 . Fig. 10 shows the computed density contours in the straight part of the nozzle for the range of $\phi=2.2$ to 3.02. The results confirm the formation of shock wave in the straight part of the nozzle. Some normal shocks are also formed due to the sudden rise in temperature across the shock as explained in the previous works ${ }^{(15,16)}$. During the startup transient of supersonic nozzle flow, at pressure ratio $\phi=2.2$, the oblique shock wave (first shock) is located just at the beginning of the straight part of the nozzle. As $\phi$ increases to 3.02 , the first shock wave moves downstream with stronger magnitude. At $\phi=3.02$ which corresponds to the final steady state in the startup transient, the computed flow field is nearly the same to that employed as the initial conditions in the shutdown transient. In the shutdown transient, as $\phi$ decreases again to 2.2 , the shock wave moves upstream and the strength seems to be stronger than those found in the startup transient. From a series of computations, it is found that the location and strength of the first shock wave is significantly different in the processes of the startup and shutdown transients. This clearly revealed that there exit a hysteretic behavior in the formation of shock wave in the straight part of the supersonic nozzle for the range of pressure ratio $\phi=2.2$ to 3.02 .

More quantitative data for hysteresis phenomena

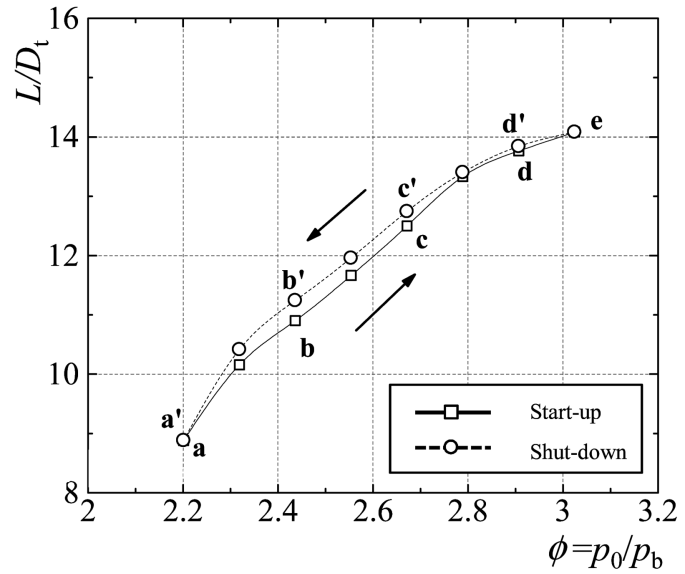

Fig. 11. Hysteretic behavior at location of shock wave

is presented in Fig. 11, where the location of first shock wave $L / D_{\mathrm{t}}$, measured from the nozzle throat, in the straight part of the nozzle during the processes of shutdown and startup transients are plotted against pressure ratio $\phi$. In the process of the startup transient, the position of the first shock moves downstream with an increase in $\phi$. On the contrary, in the process of the shutdown transient, the shock position moves upstream with a decrease in $\phi$. Hysteresis loop in the location of shock wave is found in both the transient processes.

Fig. 12 shows the hysteretic behavior in the shock strength which is corresponds to the static pressures $p / p_{\mathrm{b}}$ behind the shock. In the process of

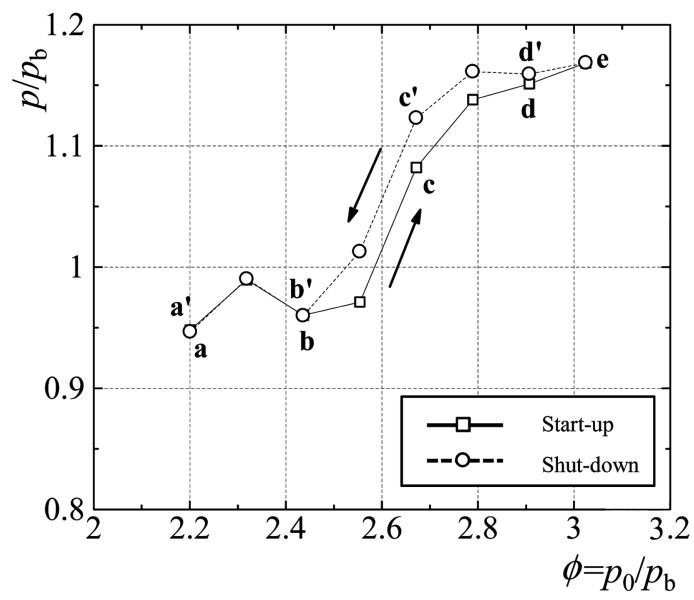

Fig. 12. Hysteretic behavior in shock strength 
the startup transient, the strength of the first shock increase with an increase in $\phi$. On the contrary, in the process of the shutdown transient, the shock strength decreases with a decrease in $\phi$. Therefore, the hysteretic behavior is produced during both the processes of the startup and shutdown transients.

\section{Conclusion}

Experimental and computational studies were carried out to investigate the hysteretic behavior in the formation of shock waves in supersonic nozzle. The axisymmetric, unsteady, compressible NavierStokes equations were solved numerically to simulate the flow field concerned with the hysteretic behavior during the processes of the shutdown and startup transients of supersonic nozzle flow. Hysteresis phenomena for the location of shock wave in a supersonic nozzle were investigated experimentally. As per the results, hysteresis phenomenon for shock wave in the Laval nozzle was confirmed at a certain specific condition. The relationship between hysteresis phenomenon and the range of the rate of change of pressure ratio with time was shown experimentally. The existence of hysteretic behavior in the formation, both the location and strength, of shock wave in the straight part of the supersonic nozzle within a range of pressure ratio has also been confirmed numerically.

\section{References}

1) Katanoda, H., Miyazato, Y., Masuda, M. and Matsuo, K., 2000, "Pitot Pressures of CorrectlyExpanded and Underexpanded Free Jets from Axisymmetric Supersonic Nozzles," Shock Waves, Vol.10, No.3, pp.95-101.

2) Yüceil, K. B. and Ötügen, M. V., 2002, "Scaling Parameters for Underexpanded Supersonic Jets," Physics of Fluids, Vol.14, No.12, pp.4206-4215.

3) Teshima, K., 1993, "Shock Structure of a Supersonic Free Jet Issuing from a Rectangular Orifice," Proc. Symp. on Shock Waves, Japan' 92, pp.473-476.

4) Chpoun, A. and Ben-Dor, G., 1995, "Numerical Confirmation of the Hysteresis Phenomenon in the Regular to the Mach Reflection Transition in Steady
Flows," Shock Waves, Vol.5, pp.199-203.

5) Ben-Dor, G., 1995, "Reconsideration of the State-ofthe-Art of the Shock Wave Reflection Phenomenon in Steady Slows," Jap. Soc. of Mech. Eng., Int. Journal, B, Vol.38, No.3, pp.325-334.

6) Ivanov, M. S., Vandromme, D., Fomin, V. M., Kudryavtsev, A. N., Hadjadj, A. and Khotyanovsky, D. V., 2001, "Transition between Regular and Mach Reflection of Shock Waves: New Numerical and Experimental Results," Shock Waves, Vol.11, pp.199207.

7) Ben-Dor, G., Elperin, T., Li, H. and Vasiliev, E., 1997, "Downstream Pressure Induced Hysteresis in the Regular $\leftrightarrow$ Mach Reflection Transition in Steady Flows," Phys. Fluids, Vol.9, No.10, pp.30963098.

8) Ben-Dor, G., Ivanov, M., Vasilev, E. I. and Elperin, T., 2002, "Hysteresis Processes in the Regular Reflection $\leftrightarrow$ Mach Reflection Transition in Steady Flows," Progress in Aerospace Sciences, Vol.38, pp.347-387.

9) Gribben, B. J., Badcook, K. J. and Richards, B. E., 2000, "Numerical Study of Shock-Reflection Hysteresis in an Underexpanded Jet," AIAA Journal, Vol.38, No.2, pp.275-283.

10) Hadjadj, A., 2004, "Numerical Investigation of ShockReflection Phenomena in Overexpanded Supersonic Jet," AIAA Journal, Vol.42, No.3, pp.570-577.

11) Baig, A., Rizvi, H., Zahir, S. and Khan, M. A., 2005, "Investigation of Shock-Reflection Hysteresis Pheno mena in Supersonic Jet of Overexpanded Flow", Proceedings of the 8th International Symposium on Fluid Control Measurement and Visualization, Chengdu, China, pp.1-9.

12) Yasunobu, T., Matsuoka, K., Kashimura, H., Matsuo, $S$. and Setoguchi, T., 2006, "Numerical Study for Hysteresis Phenomena of Shock Wave Reflection in Overexpanded Axisymmetric Supersonic Jet," Journal of Thermal Science, Vol.15, No.3, pp.220-225.

13) Irie, T., Yasunobu, T., Kashimura, H. and Setoguchi, T., 2004, "Hysteresis Phenomena of Mach Disk Formation in an Underexpanded Jet," Theoretical and Applied Mechanics Japan, Vol.53, pp.181-187.

14) Kim, H. D., Kang, M. S., Otobe, Y. and Setoguchi, T., 2009, "The Effect of Nonequilibrium Condensation on Hysteresis Phenomenon of Under-Expanded Jets," 
Journal of Mechanical Science and Technology, Vol.23, pp.856-867.

15) Al-Hasan, N. S. and Schnerr, G. H., 2007, "Aerodynamic Optimization of Laval Nozzle Flow with Shocks: Numerical Investigation of Active/Passive Shock Control Via Expansion Fans," PAMM - Proc. Appl. Math. Mech., 7, 4110003-4110004.

16) Matsuo, K., Miyazato, Y. and Kim, H. D., 1999, "Shock Train and Pseudo-Shock Phenomena in Internal Gas Flows," Progress in Aerospace Sciences, Vol.35, pp.33-100.

17) Menter, F. R., 1993, "Zonal Two Equation $k$-̀̀ Turbulence Models for Aerodynamic Flows," AIAA
Paper 93-2906.

18) Menter, F. R., 1994, "Two-Equation Eddy-Viscosity Turbulence Models for Engineering Applications," AIAA Journal, Vol.32, pp.269-289.

19) Menter, F. R., Kuntz, M. and Langtry, R., 2003, "Ten Years of Industrial Experience with the SST Turbulence Model," Turbulence, Heat and Mass Transfer, 4, pp.625-632.

20) Nebbache, A. and Pilinski, C., 2006, "Pulsatory Phenomenon in a Thrust Optimized Contour Nozzle," Aerospace Science and Technology, Vol.10, pp.295-308. 